Association for Information Systems AIS Electronic Library (AISeL)

Wirtschaftsinformatik Proceedings 1999

Wirtschaftsinformatik

February 1999

\title{
Investitionen in Standardsoftware: Ein geschäftsorientierter Ansatz zur Nutzenmessung und -bewertung
}

Sebastian Muschter

IMG Americas Inc., Philadelphia, USA, Sebastian_Muschter@img-a.com

Hubert Österle

Universität St. Gallen, hubert.oesterle@unisg.ch

Follow this and additional works at: http://aisel.aisnet.org/wi1999

\section{Recommended Citation}

Muschter, Sebastian and Österle, Hubert, "Investitionen in Standardsoftware: Ein geschäftsorientierter Ansatz zur Nutzenmessung und -bewertung" (1999). Wirtschaftsinformatik Proceedings 1999. 24.

http://aisel.aisnet.org/wi1999/24

This material is brought to you by the Wirtschaftsinformatik at AIS Electronic Library (AISeL). It has been accepted for inclusion in Wirtschaftsinformatik Proceedings 1999 by an authorized administrator of AIS Electronic Library (AISeL). For more information, please contact elibrary@aisnet.org. 


\section{Investitionen in Standardsoftware: Ein geschäftsorientierter Ansatz zur Nutzenmessung und -bewertung}

Sebastian Muschter

IMG Americas Inc., Philadelphia, USA (Sebastian_Muschter@img-a.com)

Hubert Österle

Universität St. Gallen (uuert.Oesterle@unisg.ch)

\section{Inhalt}

1 Einführung

2 Zur Ausschöpfung der Nutzenpotentiale bei der Einführung von Standardsoftware

3 Nutzenmanagement für IS-Investitionen - eine Bestandsaufnahme

3.1 Generelle Ansätze

3.2 Analysen zur "Total Cost of Ownership (TCO)"

3.3 Benchmarking im Informationsmanagement

3.4 Nutzenmanagement in der Praxis

3.5 Fazit: Defizite bestehender Ansätze

4 Ein geschäftsorientierter Ansatz zum Nutzenmanagement

4.1 Komponenten des geschäftsorientierten Nutzenmanagements

4.2 Prozeßkennzahlen

4.3 Meßsysteme

4.4 Prozeßbenchmarks

4.5 Geschäftsnutzen

5 Zusammenfassung und Ausblick 


\begin{abstract}
Unternehmen verknüpfen vermehrt die Umgestaltung von Geschäftsprozessen mit der Einführung betriebswirtschaftlicher Standardsoftware. Derartige Projekte erweisen sich als kostspielig und komplex - mit der Konsequenz, daß Verbesserungspotentiale ungenutzt bleiben.
\end{abstract}

Zur Lösung bieten sich zwar Ansätze des sog. (IS/IT-)Nutzenmanagements an, allerdings sind die Beurteilungskriterien bestehender Ansätze nur ungenügend an den geschäftlichen Zielsetzungen der Investition orientiert. Konzepte zur Bestimmung der Potentiale und der Zielerreichung auf der geschäftlichen Ebene fehlen.

Darauf aufbauend umreißt der Artikel eine neue, "geschäftsorientierte" Methode zum Nutzenmanagement; sie mißt den Erfolg der IS-Investition an den damit verbundenen Prozeßverbesserungen. Komponenten der Methode sind Prozeßkennzahlen, Meßsysteme und Benchmarks.

\title{
1 Einführung
}

Business Engineering, die grundlegende Neugestaltung von Unternehmen und ihrer Prozesse unter Zuhilfenahme von Informationstechnik und Informationssystemen (IT/IS), ist immer noch beherrschendes Thema und größte Herausforderung für die Unternehmen. Mehr denn je ist eine wesentliche Determinante für langfristigen Unternehmenserfolg das fortwährende Ändern der eigenen Geschäftspraktiken, und zwar sowohl in inkrementellen als auch in revolutionären Schritten (Tushman/O'Reilly 1996, S. 24).

Viele Unternehmen sehen in betriebswirtschaftlicher Standardsoftware (SSW) ein geeignetes Instrument, um schlanke und flexible Prozesse in ihre Organisation einzuziehen und unternehmensweit im Sinne eines "Enterprise Resource Planning (ERP)" zu koordinieren. Entsprechend steigt die Verbreitung von Standardsoftware (s. nächsten Abschnitt). In Unternehmen, die mit ihrer IT eine hohe Effizienz und Effektivität erzielen, beträgt der Anteil von SSW an den gesamten Software-Implementierungskosten bereits 75\% - im Vergleich zu 42\% bei Unternehmen mit unterdurchschnittlicher IT (Kempis/Ringbeck 1998, S. 148).

Die Kunden haben hohe Erwartungen in die SSW-Projekte; beispielsweise will die Firma Microsoft mit einem SSW-gestützten Beschaffungsprozeß Einsparungen von jährlich 12 Mio. Dollar nur durch Ausschöpfung von Lieferantenskonti erzielen. Dafür entstehen dem Unternehmen Softwarekosten von 25 Mio. Dollar (Martin 1998, S. 95). Die Lizenzkosten machen jedoch nur einen kleinen Teil der gesamten Projektkosten aus; Aufwendungen für externe Beratung, Hardware, internes Personal und Schulungen übersteigen die eigentlichen 
Lizenzkosten regelmäßig (Buxmann/König 1996, S. 163) - teilweise um den Faktor 10 (Martin 1998, S. 96; Edmondson 1997, S. 46). Für die Unternehmen resultieren daraus Projektkosten in zum Teil dreistelliger Millionenhöhe - für Owens Corning, ein amerikanischer Produzent von Baustoffen, z.B. 110 Mio. Dollar bei einem Jahresumsatz von 4 Mrd. Dollar 1996 (Stewart 1997, S. 82).

Was erhalten die Unternehmen dafür? Lassen sich die ehrgeizigen Zielsetzungen verwirklichen? Mehr und mehr Unternehmen kommen mit ihren Reengineeringund Standardsoftware-Projekten in eine Phase, in der erstmals Erträge der Investitionen eintreten sollen - ohne daß dies im erwarteten Ausmaß geschieht. "It's an open secret that the ways to fail at ERP implementation ... outnumber the ways to succeed" (Martin 1998, S. 96). Vielmehr entdecken die Unternehmen, daß sie mit der Einführung der neuen Prozesse und Systeme nur einen geringen Teil der möglichen Verbesserungen ausgeschöpft haben - wie der folgende Abschnitt 2 zeigt - und starten Projekte zur weiteren Prozeß- und Systemoptimierung.

Vor diesem Hintergrund entsteht Bedarf für methodische Konzepte, die zur Ausschöpfung der Nutzenpotentiale beitragen, die durch Investitionen in Informationssysteme entstehen. Der entsprechende Forschungsbereich wird hier analog zu Ward als IS-/IT-Nutzenmanagement bezeichnet und definiert als "the process of organizing and managing such that potential benefits arising from the use of IT are actually realized" (Ward et al. 1996, S. 214). Eine Bestandsaufnahme des Nutzenmanagements in Abschnitt 3 zeigt, daß bisherige Methoden zur Nutzenbewertung technische Verbesserungspotentiale der neuen IS-Lösungen in den Mittelpunkt stellen. Standardsoftware dient jedoch im Regelfall der Umsetzung neuer Geschäftsprozesse, ohne daß eine Trennung zwischen organisatorischer Umgestaltung und Softwareeinführung möglich wäre. Nutzenmanagement sollte entsprechend beides als Einheit betrachten und am Zusammenspiel von Prozessen und Informationssystemen ansetzen. Den Nutzen einer Investition in Standardsoftware an erzielten Prozeßverbesserungen zu messen, wird hier als "geschäftsorientierter" Ansatz bezeichnet. Dessen Komponenten beleuchtet Abschnitt 4 genauer. Ein Fallbeispiel in Abschnitt 5 rundet die Betrachtung ab.

Konzepte, die das Zusammenspiel von Prozeß und Informationssystem verbessern wollen, sind dem Forschungsgebiet der Prozeßoptimierung zuzurechnen. Allgemein fehlt diesem bisher eine hinreichende Operationalisierung (Horváth 1997, S. 114); dies gilt um so mehr für Geschäftsprozesse, die von Standardsoftware unterstützt werden - für diese sind gar keine Optimierungsmethoden bekannt (Buxmann/König 1996, S. 168). Die vorliegende Arbeit beschreibt einen Teil einer solchen Methode zur Optimierung IS- oder SSW-gestützter Prozesse. Sie entstand im Rahmen des Forschungsprojekts "Process Measurement and Benchmarking", das am Institut für Wirtschaftsinformatik der Universität St. Gallen mit zahlreichen Anwendern von Standardsoftware durchgeführt wurde. 


\section{Zur Ausschöpfung der Nutzenpotentiale bei der Einführung von Standardsoftware}

Schon längere Zeit verknüpfte sich mit zunehmenden Investitionen in Informationstechnologie der Wunsch, einen geschäftlichen Vorteil aus diesen nachzuweisen (s. Abschnitt 3). Für Investitionen in Standardsoftware gilt dies in verstärktem Maße: Immer mehr Unternehmen setzen Standardsoftware ein, die Investitionssummen sind erheblich. Zudem sind die Projekte zumeist sehr umfassend, weil Standardsoftware häufig als Katalysator des unternehmerischen Wandels dient.

Der Markt für Standardsoftware wächst beträchtlich. Bereits 20.000 Unternehmen setzen ein System der vier größten Anbieter SAP AG, Oracle, Peoplesoft und Baan Company ein; das Volumen im Markt für ERP-Systeme betrug nach einem Wachstum von 40\% ca. 10 Mrd. Dollar im Jahre 1997 (Martin 1998, S. 95). Im Jahre 2000 soll das Marktvolumen bereits 23 Mrd. Dollar ausmachen (Benchmarking Partners 1997a, S. 3). Die Marktführer wachsen dabei sogar noch schneller: Die Umsätze der vier größten Anbieter stiegen 1997 sogar zwischen 56\% und 81\% (Martin 1998, S. 95; SAP 1998a; o.V. 1998; Rieker 1998). Das System R/3 der SAP AG gilt als die umfassendste ERP-Lösung (Benchmarking Partners 1997b, S. 16), es besitzt einen Anteil von 36\% im Markt der ERP-Systeme und ist damit deutlicher Marktführer. Insgesamt zählen über 7500 Unternehmen zu den SAP-Kunden (SAP 1998b), alleine in Deutschland setzen 95 der 100 größten Unternehmen SAP-Systeme ein (Rieker 1998, S. 116).

Die Unternehmen streben im Rahmen der SSW-Einführung nicht nur Veränderungen am Informationssystem an, sondern verändern auch ihre Organisation. Der gleichzeitige Wandel führt zu kostspieligen und komplexen Projekten (Davenport 1998, S. 122). Die Projektkosten belaufen sich im Durchschnitt auf $1 \%$ bis 1,5\% des Jahresumsatzes und absorbieren damit $50 \%$ bis 75\% des gesamten IS/IT-Budgets der Unternehmen (Computer Finance 1997, S. 3). Die Komplexität der Projekte ist das meist genannte Problem bei der R/3Einführung (Knolmayer et al. 1997). Es ergibt sich zum einen aus der Bandbreite der Projekte, die im Regelfall mehrere Prozeßbereiche und einen großen Teil des Informationssystems berühren, und zum anderen aus den zahlreichen Änderungen an Prozessen und Systemen, die die Projekte nach sich ziehen.

Zahlen aus einer Studie des Jahres 1995 über die Einführung von SAP-Systemen belegen dies: Um die Integrationspotentiale eines SAP-Systems auszunutzen, umfassen die Installationen fast immer mehr als ein Modul; laut der Studie setzen sogar mehr als $75 \%$ der 220 damals befragten europäischen R/3- und R/2Nutzer vier oder mehr Module ein. Die gleiche Studie zeigte: Nur 20\% der Projekte ließen die Geschäftsprozesse unverändert, vielfach wurde die SSWEinführung zum Auslöser eines Reengineering. Nur bei 12\% der Projekte unterblieben Erweiterungen oder Modifikationen an der Software, womit die 
Kunden die (im Releasestand von 1995) unzureichende Abdeckung ihrer Anforderungen überwinden wollten. Insbesondere die Modifikationen führten im Schnitt zu einer deutlichen Steigerung von Projektdauer und -kosten, weswegen sich viele Anwender im Nachhinein auf weniger einschneidende Änderungen beschränken würden (Buxmann/König 1996; Buxmann/König 1997).

Der Herausforderung, organisatorischen Wandel mit einer Veränderung an den Informationssystemen zu verknüpfen, begegnen die Unternehmen heute auf unterschiedliche Weise. Im Regelfall verfolgen die Kunden bei der SSWEinführung in Grundzügen eine von zwei Normstrategien, die in Abb. 1 illustriert sind (Becker et al. 1997; S. 6f., Dolmetsch et al. 1998, S. 5ff.): In der ersten Variante entwirft ein Unternehmen zunächst seine Geschäftsprozesse und implementiert diese danach in der Standardsoftware. Im Mittelpunkt der Projekte steht die Neugestaltung der Prozesse, weswegen diese Variante auch als prozeßorientierter Ansatz bezeichnet wird. In der zweiten Variante implementiert das Unternehmen die Standardsoftware zügig und prototypisch, ohne im Vorfeld die Prozesse systematisch neu zu entwerfen. Die spätere Reorganisation erfolgt auf Basis des neuen Informationssystems. Dieser Ansatz wird auch als "Implement and Tune" (Forrester 1996) oder als informationssystemorientierter Ansatz bezeichnet.

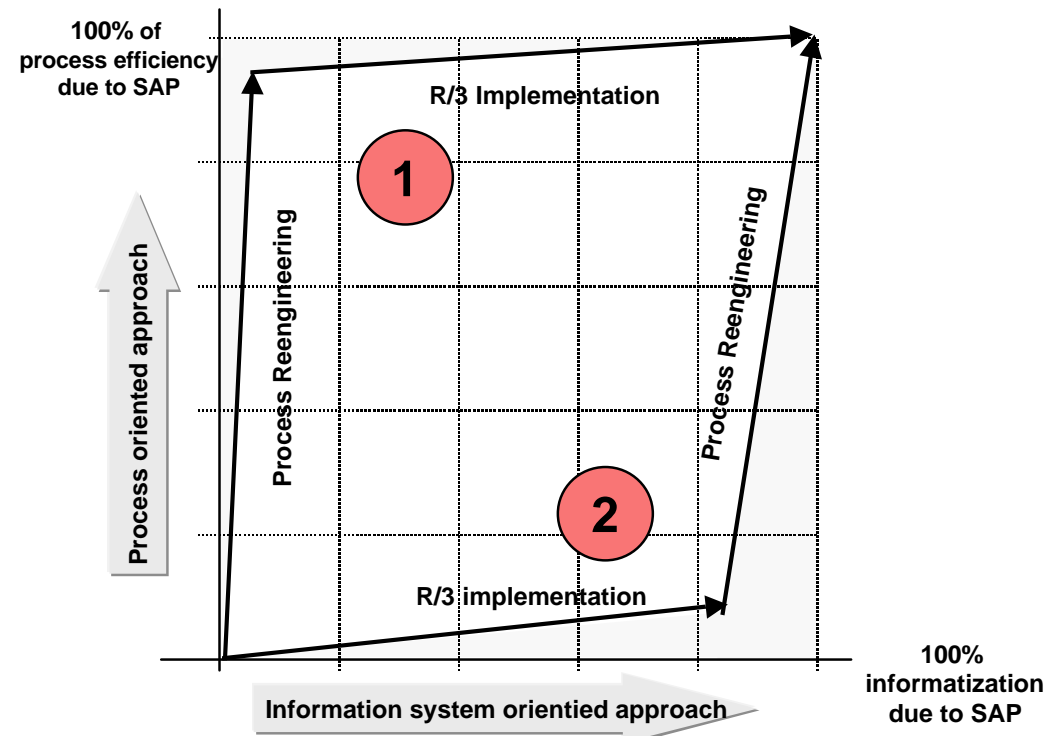

Abbildung 1: Normstrategien bei der Einführung von Standardsoftware (Becker et al. 1997, S. 7)

Inwieweit erreichen Unternehmen die Zielsetzungen, die sie mit der Einführung von Standardsoftware verknüpfen? Empirische Untersuchungen zu dieser Frage- 
stellung fehlen bislang; die folgenden Erkenntnisse gründen auf Erfahrungen, die im Rahmen des Forschungsprojekts "Process Measurement \& Benchmarking" gemeinsam mit den Projektpartnern entstanden. Sie zeigen: Unabhängig von der Einführungsstrategie sind die Verbesserungspotentiale nach Inbetriebnahme der neuen Systeme erheblich.

Als Beispiel für ein Projekt, das Standardsoftware-Einführung mit Reengineering verknüpfte, mag hier ein Projekt bei der Ciba Specialty Chemicals dienen. Ciba hatte 1993 begonnen, grundlegend neue Logistikprozesse zu entwerfen, um deutliche Verbesserungen hinsichtlich Lieferpünktlichkeit oder Wunschliefertreue zu erreichen (Bäckert/Packowski 1997). Anschließend setzte Ciba die Konzepte in der Software SAP R/3 um. Prozeßmessungen nach Inbetriebnahme der neuen Systeme zeigten, daß Ciba nur die Hälfte der möglichen Verbesserungen direkt erreicht hatte - Abb. 2 zeigt die Prozentsätze der zum Wunsch- bzw. bestätigten Termin ausgelieferten Aufträge. Die andere Hälfte erbrachte erst die anschlieBende kontinuierliche Verbesserung. In den Worten des Projektleiters: "Going live is half the journey" (Bäckert/Brecht 1996).

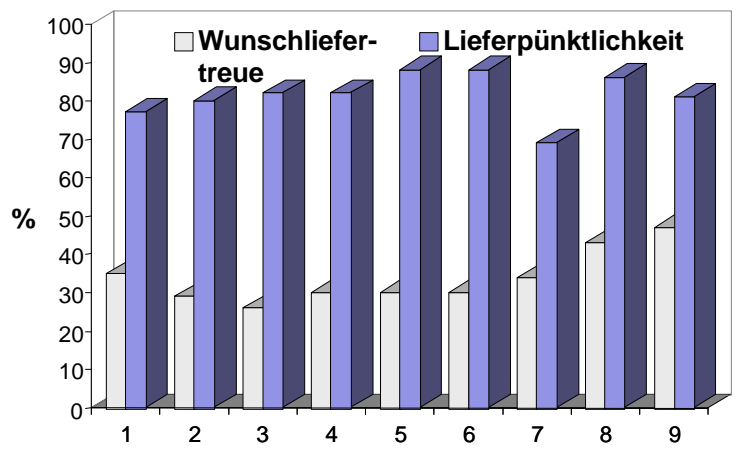

Abbildung 2: Wunschliefertreue und Lieferpünktlichkeit nach Reengineering und Inbetriebnahme der neuen Systeme (Bäckert/Brecht 1996)

Ein anderes, anonymisiertes Beispiel entstammt einem Unternehmen, das die größten Nutzenpotentiale erst nach Inbetriebnahme der neuen Systeme realisieren möchte und zu diesem Zweck u.a. ein regelmäßiges externes Prozeßbenchmarking durchführt. Abb. 3 zeigt für ein Werk dieses Unternehmens die Fortschritte in der Lieferpünktlichkeit, die die Messungen für die Zeit nach der Inbetriebnahme auswiesen.

Wo liegen die Gründe für die erheblichen Verbesserungspotentiale? Bei Verfolgung der Variante 1, die die Einführung mit einem vorherigen Reengineering verknüpft, gelingt die Überführung von Prozeßkonzepten in die Standardsoftware selten zu $100 \%$. Dies liegt zum Teil an vorher unbekannten Grenzen der neuen Software oder an den langen Zeiträumen zwischen Prozeßentwurf und Umsetzung, die einen Teil der Konzepte obsolet werden lassen. Der Hauptgrund liegt 
jedoch in der hohen Komplexität eines gleichzeitigen Wandels von Organisation und Informationssystem. So müssen die Projektteams häufig angesichts des wachsenden Termindrucks Abstriche und Verzögerungen bei den geplanten Prozeßverbesserungen hinnehmen, um überhaupt termingerecht ein lauffähiges System zu erreichen. Die Ausschöpfung dieser Potentiale bleibt der Zeit nach dem Produktivstart vorbehalten.

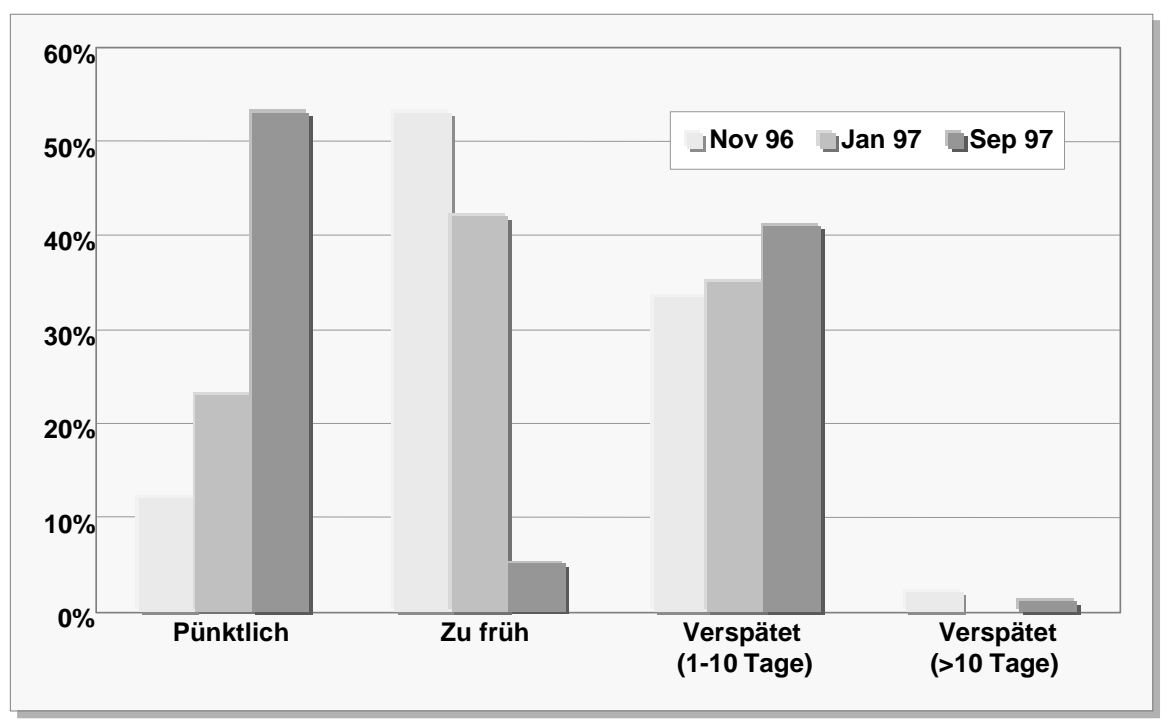

Abbildung 3: Entwicklung der Lieferpünktlichkeit nach einer StandardsoftwareEinführung ohne vorheriges Reengineering

Eine Einführung nach der Variante 2 verschiebt die Realisierung der größten Nutzenpotentiale bewußt auf die Zeit nach der Inbetriebnahme. Das schnell zur Verfügung gestellte Informationssystem wird zum Katalysator des nachfolgenden Verbesserungsprozesses, weil es über Prozeßmessungen Transparenz schafft und Prozesse im gesamten Unternehmen standardisiert (Groenfeldt 1997, S. 40). Das Verbesserungspotential ist zumeist deswegen erheblich, weil im Zuge der Einführung nur minimale und einfache Änderungen an den bestehenden Geschäftsprozessen vorgenommen werden. Auch wird die neue Software in der Einführungsphase erst in Grundzügen erschlossen, was später Potentiale durch die Einbindung weiterer Funktionalität eröffnet.

Als Fazit lassen sich aus diesen kurzen Beispielen die Erkenntnisse gewinnen, $\mathrm{da} ß$ Standardsoftware-Einführungen im Regelfall mit organisatorischem Wandel verknüpft sind und angesichts dieser Komplexität unabhängig von der gewählten Einführungsstrategie ein erhebliches Verbesserungspotential nach Inbetriebnahme der neuen Systeme besteht. Daraus leitet sich der Bedarf nach einer Methode zum Nutzenmanagement ab, die diesem Umfeld gerecht wird. 


\section{Nutzenmanagement für IS-Investitionen - eine Bestandsaufnahme}

Die Ansätze des IS/IT-Nutzenmanagements entstanden als Reaktion auf das sogenannte Produktivitätsparadoxon, wonach aus Investitionen in IS/IT nicht in jedem Fall die erwarteten Produktivitätssteigerungen resultierten (Jurison 1997, S. 33). Einen Überblick über die verschiedenen Studien in diesem Bereich gibt (Potthof 1998).

\subsection{Generelle Ansätze}

Der potentielle Nutzen eines Informationssystems wird nach Farbey et al. vom Ausmaß des unternehmerischen Wandels bestimmt, der mit der Implementierung des Informationssystems beabsichtigt ist bzw. mit der Implementierung einhergeht (Farbey et al. 1995). Die Autoren unterscheiden dafür in einem Modell, das sie "Stufenleiterkonzept zur Nutzenbewertung" nennen, acht Stufen, in die sie IS-Projekte nach dem Grad des angestrebten Wandels einordnen: Ein Projekt, das nur der Überwindung des Jahr-2000-Problems dient, fällt in die Stufe 1 "Erzwungene Anpassungen"; die Einführung einer neuen IS-Architektur, die neue Prozesse nach einem unternehmensweiten Reengineering unterstützen soll, ordnet sich als Projekt in die Stufe 8 "Transformation des Unternehmens" ein.

Gleichzeitig empfehlen die Autoren für jede Stufe spezielle Verfahren zur Nutzenmessung und -bewertung, die den unterschiedlichen Risiken und Erträgen der Projekte Rechnung tragen. Je umfassender der beabsichtigte Wandel, desto höher ist das Risiko, mit der Applikation den geplanten Nutzen nicht zu erreichen, desto höher ist aber auch der potentielle Ertrag. Tabelle 1 zeigt die Stufen des Wandels und die zugehörigen Projekttypen, den angestrebten Projektnutzen sowie mögliche Verfahren der Nutzenmessung.

Eine Nutzenmessung wird um so schwieriger, je umfassender die Zielsetzungen angelegt sind, die ein Unternehmen mit einem IS-Projekt erreichen möchte; schon weil die positiven Effekte einer IS-Investition auf höheren Stufen schwerer von anderen Einflußfaktoren zu trennen sind (Dempsey et al. 1998, S. 127). Gleichzeitig nimmt aber auch die Bedeutung der Nutzenmessung von Stufe zu Stufe zu, da mit dem Projektrisiko und der Höhe des potentiellen Ertrages auch die Wahrscheinlichkeit ansteigt, die bestehenden Nutzenpotentiale nicht vollständig auszuschöpfen. 


\begin{tabular}{|c|c|c|c|}
\hline Stufe & Beschreibung & Nutzen/Projektziel & $\begin{array}{l}\text { Verfahren zur } \\
\text { Nutzenmessung (Bsp.) }\end{array}$ \\
\hline 8 & $\begin{array}{l}\text { Business } \\
\text { Transformation }\end{array}$ & $\begin{array}{l}\text { Strategische Neuausrichtung } \\
\text { und Verbesserung des } \\
\text { gesamten Unternehmens }\end{array}$ & $\begin{array}{l}\text { Isolierung des Beitrags der } \\
\text { IT zum Gesamtnutzen } \\
\text { meist nicht möglich }\end{array}$ \\
\hline 7 & $\begin{array}{l}\text { Strategic } \\
\text { systems }\end{array}$ & $\begin{array}{l}\text { Stärkung der } \\
\text { Wettbewerbsposition }\end{array}$ & $\begin{array}{l}\text { Umfassende Beurteilung } \\
\text { des verbesserten } \\
\text { Geschäftsumfelds } \\
\text { (schwierig); dazu ROI- } \\
\text { Analysen }\end{array}$ \\
\hline 6 & $\begin{array}{l}\text { Interorganization } \\
\text { al systems }\end{array}$ & $\begin{array}{l}\text { Unternehmensübergreifend } \\
\text { gesteigerte Effizienz und } \\
\text { Effektivität }\end{array}$ & $\begin{array}{l}\text { Abschätzen des } \\
\text { kombinierten Nutzens aller } \\
\text { Partner }\end{array}$ \\
\hline 5 & Infrastructure & $\begin{array}{l}\text { Ermöglichung zukünftiger } \\
\text { IS-Aktivitäten }\end{array}$ & $\begin{array}{l}\text { Bewertung des Nutzens der } \\
\text { Projekte, die Infrastruktur } \\
\text { ermöglichte }\end{array}$ \\
\hline 4 & MIS and DSS & $\begin{array}{l}\text { Höhere Manager-Produktivität } \\
\text { durch " bessere" Information }\end{array}$ & $\begin{array}{l}\text { Managerbefragungen; } \\
\text { Untersuchung, wieviel } \\
\text { "Geld" Manager für } \\
\text { bessere Information zu } \\
\text { zahlen bereit sind }\end{array}$ \\
\hline 3 & $\begin{array}{l}\text { Direct value } \\
\text { added systems }\end{array}$ & $\begin{array}{l}\text { Bessere Produkte und } \\
\text { Serviceleistungen }\end{array}$ & $\begin{array}{l}\text { Kundenbefragungen, } \mathrm{ROI}- \\
\text { Analysen }\end{array}$ \\
\hline 2 & Automation & Höhere Effizienz & $\begin{array}{l}\text { Messung der } \\
\text { Produktivitätssteigerung }\end{array}$ \\
\hline 1 & $\begin{array}{l}\text { Mandatory } \\
\text { changes }\end{array}$ & $\begin{array}{l}\text { Lauffähiges System bei } \\
\text { geringen } \\
\text { Implementierungskosten }\end{array}$ & $\begin{array}{l}\text { Berechnung der } \\
\text { Implementierungskosten }\end{array}$ \\
\hline
\end{tabular}

Tabelle 1: Benefits' Evaluation Ladder (Farbey et al. 1995)

Konkrete Verfahren zur Nutzenmessung, insbesondere für Projekte der höheren Stufen, beschreiben die Autoren nicht: "Movement up the ladder changes the emphasis from precise quantification to more judgemental evaluation styles" (Farbey et al. 1995, S. 49). Damit reagieren sie auf die Notwendigkeit, bei ambitiöseren Projekten auch den qualitativen, nicht-finanziellen Nutzen in die Projektbewertung einzubeziehen: "Conventional cost/benefit and return on investment (ROI) methods are rarely suitable for reaching decisions on strategic investments. Indeed some of the systems now seen to confer strategic benefits would have failed any test based on cost/benefit and ROI methods" (Farbey et al. 1995, S. 48). Die traditionellen Bewertungsansätze eignen sich nicht zur Messung der wichtigsten Nutzenpotentiale, die beispielsweise in höherer Kundenzufriedenheit, einem höheren Service-Level, höherer Mitarbeiterzufriedenheit oder höherer Produktqualität liegen (Apostolopoulos/Pramataris 1997, S. 296). Dies erklärt nach Jurison auch einen Teil des Produktivitätsparadoxons: Da herkömmliche Kennzahlen zur Produktivität die wichtigsten Nutzenpotentiale einer IS-Investition nicht fassen 
können, weisen die Zahlen folglich auch keine Produktivitätsfortschritte im Zuge des Projekts aus (Jurison 1997, S. 33).

\subsection{Analysen zur "Total Cost of Ownership (TCO)"}

Einer ähnlichen Kritik, unzureichende Beurteilungsmaßstäbe zu propagieren, sehen sich die in jüngster Zeit vermehrt diskutierten Ansätze ausgesetzt, die die Beurteilung von Informationssystemen anhand ihrer "Total Cost of Ownership (TCO)" vornehmen wollen. Die TCO sind die Gesamtkosten einer IS-Lösung über die Nutzungsdauer hinweg; sie beinhalten nicht nur die Anschaffungs-, sondern z.B. auch Verwaltungs-, Wartungs- oder Schulungskosten (Riepl 1998, S. 8). Da sie ausschließlich Kosten- und keine Ertragsapekte berücksichtigen, eignen sich TCO-Analysen zwar prinzipiell zur Senkung laufender Kosten, nicht aber als Basis einer Entscheidung zwischen verschiedenen Investitionsvarianten (Dempsey et al. 1998, S. 129). In die gleiche Richtung zielen Initiativen von Standardsoftware-Anbietern, die für ihre Software im Sinne einer "Lowest Cost of Ownership (LCO)" Unterstützung leisten bei der Auswahl und Gestaltung einer kostenoptimalen Kombination aus Software, Hardware, Betriebssystem und Datenbank (Kidler/Gümbel 1998).

Positiv wirkt sich bei TCO-Analysen zumindest die Verfügbarkeit von Vergleichszahlen und von verschiedenen, detailliert beschriebenen Berechnungsvarianten aus, auch wenn sich diese vorwiegend auf Investitionen in PC-Arbeitsplätze beziehen (s. die Übersicht bei Riepl 1998, S. 8). Für weitergehende ROIAnalysen, die nicht nur die Kostenseite einbeziehen wollen, existieren bedeutend weniger Ansätze, auch die Erträge zu quantifizieren (Changchit et al. 1998, S. 146f.; Kidler 1998, S. 26f.), zudem fokussieren sie eher auf eine Nutzenschätzung im Vorfeld der Implementierung als auf die Nutzenmessung danach (DeLone/McLean 1993, S. 10).

\subsection{Benchmarking im Informationsmanagement}

Benchmarking, nach Camp "the search for industry best practice that lead to superior performance" (Camp 1995, S. 15), bietet sich prinzipiell als Konzept an, aufgrund von Erfahrungen anderer Unternehmen mit neuen IS-Lösungen auf Nutzenpotentiale für das eigene Unternehmen zu schließen. Folgerichtig existieren einige Ansätze, die IS-Investitionen oder das Informationsmanagement insgesamt mit Hilfe von Benchmarking beurteilen wollen. Allerdings verfallen sie dem gleichen Fehler, Erfolgsmaßstäbe nicht oder nur unzureichend am erzielten Geschäftsnutzen auszurichten. Rehäuser und Krcmar unterscheiden zur Beurteilung des Informationsmanagements (IM), zu dessen Aufgaben auch das Management der Informationssysteme zählt, die Dimensionen der Effizienz und Effektivität. Die Effizienz des IM beleuchtet die Qualität der Prozesse innerhalb des Informationsmanagements; die Effektivität des IM kommt dagegen in einer guten Unterstützung der eigentlichen Geschäftsprozesse zum Ausdruck 
(Rehäuser/Krcmar 1995, S. 110). Entsprechend dieser Unterteilung konzentrieren sich die Beurteilungskriterien im Nutzenmanagement zu stark auf die Effizienz des Informationsmanagements; die Betrachtung der Effektivität kommt darüber zu kurz.

Diese Schwerpunkte werden an einem weitverbreiteten Beispiel für Benchmarking im Informationsmanagement deutlich: Die sog. "Information Technology Performance Improvement Studies" der Firma Compass vergleichen die Kosten und Leistungen des unternehmensinternen Informationsmanagements (Compass 1998). Nach einer Einordnung von Lacity und Hirschheim fallen die Benchmarks, die in Compass-Studien vorwiegend Anwendung finden, in den Bereich der "Data Center Operations Benchmarks". Sie gelten als wesentlich weiter entwickelt und damit glaubwürdiger als Benchmarks, die auf die Zufriedenheit der Anwender oder die Effektivität des IM abzielen (Lacity/Hirschheim 1994, S. 783). Zudem messen selbst die Benchmarks zur ISEffektivität eher die "wahrgenommene Kompetenz der IS-Mitarbeiter" als die Zufriedenheit der Anwender mit den geschäftlichen Vorteilen einer Applikation (Lacity/Hirschheim 1994, S. 785).

Die Autoren konstatieren insgesamt die Unzulänglichkeit solcher Benchmarks, dem Top-Management die Leistungen des eigenen Informationsmanagements zu verdeutlichen - die Benchmarks seien $\mathrm{zu}$ technisch, an den falschen Grundwerten orientiert oder durch Vergleich mit falschen Partnern entstanden (Lacity/Hirschheim 1994, S. 787ff.), wodurch sich auch ihre geringe Verbreitung zu erklären scheint (DeLone/McLean 1993, S. 7). Lacity und Hirschheim geben als erste Empfehlung für geeignetere Vergleichsmaßstäbe: "Benchmark what is important to management" (Lacity/Hirschheim1994, S.792).

\subsection{Nutzenmanagement in der Praxis}

Die Defizite bestehender Ansätze zur Nutzenbewertung spiegeln sich in der Praxis wider. Nach einer Studie von Price Waterhouse halten $80 \%$ der befragten EDV-Manager TCO-Analysen für wertlos (Riepl 1998, S. 12). Trotzdem beschränken sich die meisten Unternehmen auf die Berechnung möglicher Kosteneinsparungen und evaluieren nur unzureichend auch den eher qualitativen Nutzen von IS-Investitionen (Dempsey et al. 1998, S. 127ff.). Viele IS-Manager bestimmen Nutzenpotentiale einer solchen Investition intuitiv oder überschätzen sie bewußt, um Projektprüfungen einfacher zu bestehen (Changchit et al. 1998, S. 158). In einer Studie von Ward et al. bei 60 britischen Unternehmen gaben sogar $47 \%$ der befragten IS-Manager ein absichtliches Überschätzen zu. Trotz dieser Manipulationen erwarteten dennoch $86 \%$ der Unternehmen, nach einer ISImplementierung weitere Nutzenpotentiale zu entdecken - doch lediglich $19 \%$ verfolgten systematisch ihre Entdeckung und Ausschöpfung (Ward et al.1996, S. 221). Eine andere Studie kommt zu einem ähnlichen Ergebnis; lediglich $15 \%$ der 3200 befragten Unternehmen hätten je versucht, den Nutzen ihrer Investitionen in Technologie zu bestimmen (APICS 1996). "Post-Implementation 
review is valuable, although the number of organizations carrying out such audits is only a fraction of those that justify investments up front" (Skyrme/Amidon 1998, S. 24; ähnlich auch DeLone/McLean 1993, S. 8).

Neben der Unzulänglichkeit bestehender Ansätze mag ein weiterer Grund, warum nur so wenige Unternehmen Nutzenmanagement betreiben, darin bestehen, daß sie vielfach zu geringe Erwartungen an die IS-Projekte stellen. In der Studie von Ward äußerten sich die Befragten nämlich sehr zufrieden mit den allgemein erzielten Projektergebnissen, ihre Beurteilungsmaßstäbe waren allerdings selten am Geschäftsnutzen des Projektes orientiert: "In many cases the success criteria of project delivery ,on time, to budget, user satisfaction" was quoted, rather than measured benefits as a result of changes within the business"(Ward et al. 1996, S. 219; ähnlich auch DeLone/McLean 1993, S. 9). Zudem fehlen offenbar Methoden für ein systematisches Nutzenmanagement die Untersuchung von Ward et al. ergab, daß lediglich 5\% der Unternehmen eine diesbezügliche Methode im Einsatz hatten.

\subsection{Fazit: Defizite bestehender Ansätze}

Als Fazit weist das Nutzenmanagement in seiner bestehenden Ausformung vielfältige Defizite auf. Es soll die vollständige Ausschöpfung der vielfältigen Nutzenpotentiale sicherstellen, die sich einem Unternehmen durch Investitionen in IS bzw. IT eröffnen. Seine Beurteilungskriterien sind dennoch zumeist einseitig an möglichen Kosteneinsparungen und nur ungenügend an den geschäftlichen Zielsetzungen der Investition orientiert, Konzepte zur Bestimmung der Zielerreichung auf der geschäftlichen Ebene fehlen. Dies gilt um so mehr für IS-Projekte mit strategischen Bezügen, wie sie Investitionen in Standardsoftware darstellen.

\section{Ein geschäftsorientierter Ansatz zum Nutzen- management}

\subsection{Komponenten des geschäftsorientierten Nutzen- managements}

Ein geschäftsorientierter Ansatz zum Nutzenmanagement will die Erfolge einer IS-Investition an den Verbesserungen der Geschäftsprozesse messen, die mit Hilfe der neuen Systeme ermöglicht wurden. Aus dieser übergeordneten Zielsetzung ergeben sich verschiedene Komponenten, die in eine Methode zum Nutzenmanagement einfließen (s. Abb. 4). 


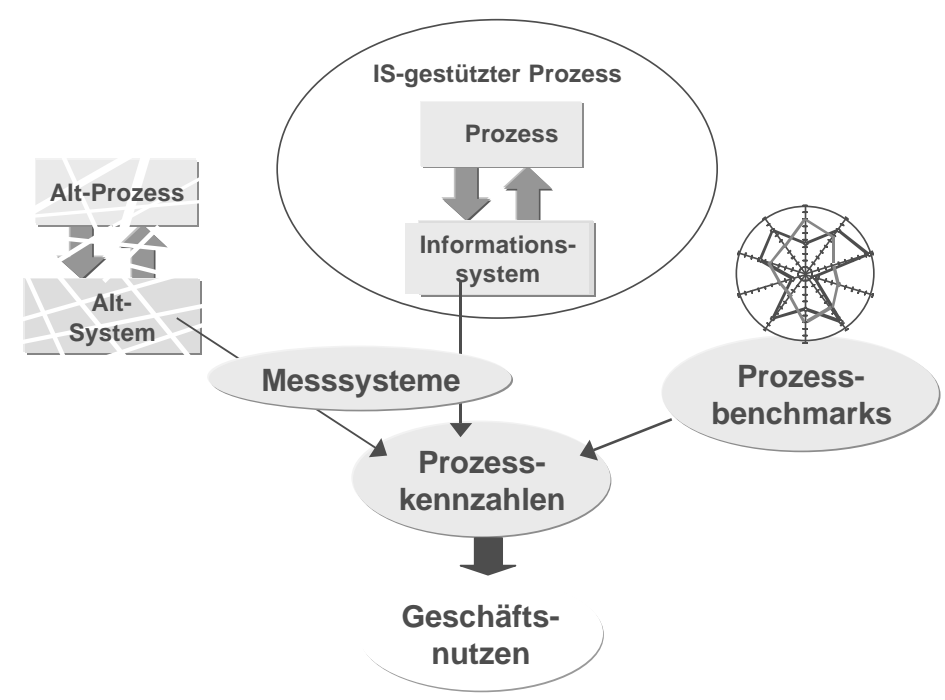

Abbildung 4: Komponenten einer Methode zum Nutzenmanagement

Betrachtungsobjekt der Methode ist ein IS-gestützter Prozeß, also die Gesamtheit aus einem Geschäftsprozeß und den (Teilen von) Datenbanken und Applikationen, die zu seiner Durchführung benötigt werden. Auf dem Informationssystem setzt ein Meßsystem auf, das Basisdaten zum Prozeß aus den betroffenen Datenbanken extrahiert und zu Prozeßkennzahlen verdichtet. Diese Kennzahlen können entweder mit Werten verglichen werden, die auf gleiche Art aus den Altsystemen gewonnen wurden und die Performance der Altprozesse widerspiegeln, oder mit externen Prozeßbenchmarks. Letztere verkörpern die als Kennzahlen ausgedrückten Leistungsstandards, die andere Unternehmen mit Prozessen auf Basis der gleichen Standardsoftware gesetzt haben. Aus dem Vergleich zwischen vorherigem, jetzigem und idealem Zustand ergeben sich der Geschäftsnutzen des Projekts und mögliche weitere Verbesserungen.

\subsection{Prozeßkennzahlen}

Die größten Nutzenpotentiale einer Investition in neue Prozesse und Systeme liegen, wie erwähnt, in Verbesserungen von Durchlaufzeiten, Mitarbeiterzufriedenheit, Service-Level usw. Folgerichtig müssen sich die Beurteilungsmaßstäbe im Nutzenmanagement genau an diesen Potentialen ausrichten - und ergeben damit direkt die Kennzahlen, mit denen die Verbesserungen ausgewiesen werden können.

Damit bilden solche prozeßorientierten Kennzahlen die Grundlage einer Messung und Bewertung des Nutzens von Informationssystemen. Schon länger besteht die Forderung an Unternehmensleitungen, solche direkten, nichtfinanziellen Führungsgrößen gleichrangig neben die finanziellen zu stellen, um 
die Leistungsfähigkeit des eigenen Unternehmens zu beurteilen (Fortuin 1988; Eccles 1991; Kaydos 1991; Kaplan/Norton 1993). Finanzielle Größen weisen verschiedene Nachteile auf: Der Erfolg eines Prozesses wird an der Kundenschnittstelle determiniert; finanzielle Größen verarbeiten diese Interaktionen indes erst mit großen Zeitverzug, nämlich wenn sie sich im Rechnungswesen niedergeschlagen haben. Weiterhin nimmt mit der zeitlichen Entfernung gleichzeitig die Zahl möglicher Einflußfaktoren zu und verringert so den Zusammenhang zwischen Kennzahl und Prozeßveränderung.

Die Ableitung eines Kennzahlensystems für einen Prozeß ist im Regelfall aufwendig, wird hier allerdings nicht im einzelnen dargelegt - für eine ausführliche Darstellung s. z.B. (Muschter 1999). Ausgangspunkt sind zumeist die Erfolgsfaktoren des Unternehmens; sie ergeben die prozeßspezifischen Erfolgsfaktoren. Letztere werden dann mit prozeßorientierten Führungsgrößen operationalisiert (s. z.B. Meyer 1994, S. 100; Weber et al. 1997, S. 441ff.). Durch diese Vorgehensweise ist sichergestellt, daß das prozeßorientierte Kennzahlensystem die Besonderheiten und strategischen Vorgaben des Prozesses reflektiert.

Im Nutzenmanagement steht jedoch weniger die kontinuierliche Weiterentwicklung des Prozesses im Vordergrund als vielmehr seine umfassende und häufig einmalige Bewertung. Hier hat sich ein standardisiertes Kennzahlensystem als geeignete Grundlage herausgestellt, das für einen Prozeß eine Sammlung häufig verwendeter Prozeßkennzahlen enthält. Es reflektiert den Umstand, daß die Faktoren Zeit, Qualität, Kosten und Flexibilität den Erfolg beinahe jedes Prozesses bestimmen; sie lassen sich immer wieder durch sehr ähnliche Kennzahlen messen (Mende 1995, S. 76).

Abb. 5 zeigt ein solches Kennzahlensystem für die Logistikprozesse Distribution, Produktion, Lagerhaltung und Beschaffung. Es entstand im Rahmen des hier zugrundeliegenden Forschungsprojekts und enthält die Kennzahlen, die sich nach Ansicht der Projektpartner für eine umfassende Beurteilung von Logistikprozessen eignen. Es zeigt vielfältige Überdeckungen zu anderen Kennzahlensystemen der Logistik, die in der Literatur beschrieben sind (s. z.B. Baumgartner 1995, S. 405; Dusch/Möller 1997; McMullan 1996, S. 89; Reichmann 1993, S. 116f.; Weber 1995, S. 47ff.).

Die Beschränkung auf wenige Führungsgrößen sorgt gleichzeitig für eine Konzentration auf die wichtigsten Prozeßverbesserungen: "A major issue concerning performance measurement is how to limit the number of indicators to a vital few and ignore the trivial many" (Bagchi 1996, S. 14). Insbesondere in der Logistik bringen weniger Kennzahlen, die über die gesamte Wertschöpfungskette erhoben werden, mehr als viele, sporadisch erhobene Größen (Boutellier 1997, S. 27). 


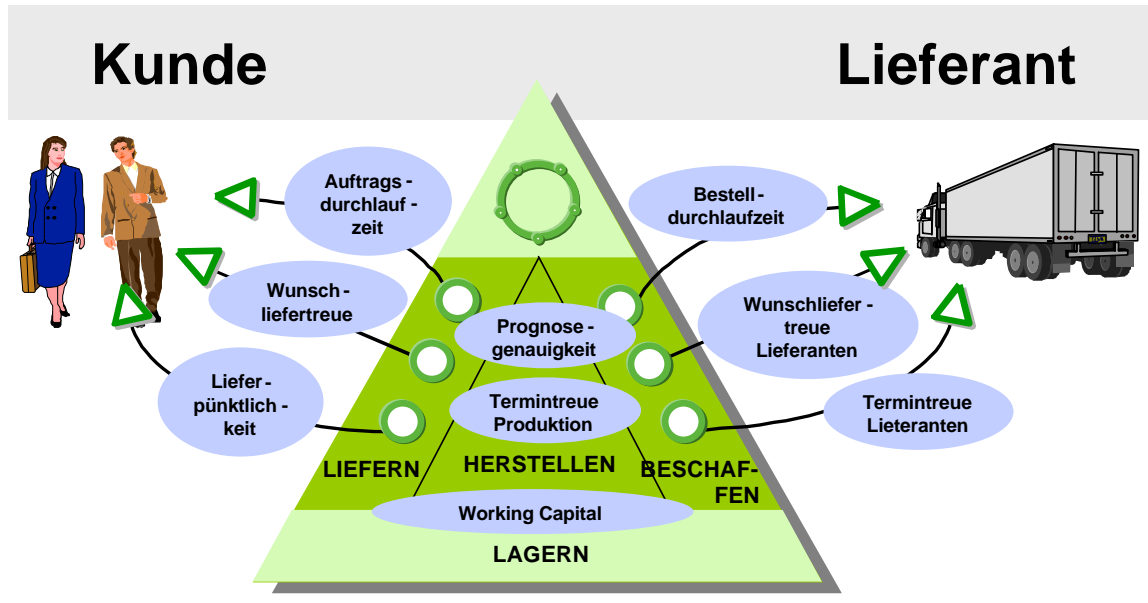

Abbildung 5: Führungsgrößen zur umfassenden Bewertung von Logistikprozessen

Die Beschränkung auf einen überschaubaren, standardisierten Kennzahlenkatalog erleichtert zudem den Aufbau einer Benchmarking-Datenbank, die eine wichtige Komponente des Nutzenmanagements darstellt (s.u.).

\subsection{Meßsysteme}

Die Auswahl von Führungsgrößen ist nur ein erster Schritt, um den Zustand eines Prozesses bewerten zu können. Ebenso wichtig ist ihre Meßbarkeit. Je intensiver sich Unternehmen mit Prozeßverbesserung beschäftigen, desto mehr entwickelt sich das Fehlen geeigneter Meßdaten über den Prozeß zu einem Haupthindernis (Grover et al. 1998, S. 56; Harrington 1991, S. 164; Millen/Maggard 1997, S. 178; New/Payne 1995, S. 74). Der Grund für die mangelnde Verfügbarkeit liegt im großen Aufwand, der für Datensammlung und -aufbereitung bei prozeßorientierten Kennzahlensystemen anfällt (Aichele/Kirsch 1995, S. 130; Aichele 1997, S. 251). Hinzu tritt die hohe Manipulierbarkeit manuell erhobener Daten (Mende 1995, S. 113; Venkatraman/Ramanujam 1986; S. 809); sie unterminiert den Zweck des Kennzahlensystems - unglaubwürdige Zahlen eignen sich nicht, den tatsächlichen Nutzen einer Prozeßumgestaltung oder eines neuen Informationssystems zu bestimmen.

Das objektive, einfache Messen von Daten gehört damit zu den wichtigsten Herausforderungen, um der Verbreitung des geschäftsorientierten Nutzenmanagements in der Praxis Vorschub zu leisten. Eine Lösung für dieses Problem bietet ein IS-gestütztes Meßsystem, das die Prozeßdaten automatisch aus den unterstützenden Informationssystemen extrahiert. Es eröffnet die Chance, Manipulierbarkeit und Erhebungsaufwand prozeßorientierter Daten zu senken. So kann z.B. der Zeitpunkt einer Auftragserfassung anhand eines DatenbankZeitstempels gemessen werden, der vom Auftragssachbearbeiter nicht beein- 
flußbar ist. Entscheidungen können auf Basis von Vollerhebungen statt auf Basis möglicherweise willkürlich zusammengestellter Stichproben gefällt werden (Nippel 1995, S. 170), mit dem Vorteil weiter gesteigerter Objektivität: "The pick-up of information for problem identification purposes can be hampered by ... screening out information that is inconsistent with expectations" (Smith 1996, S. 543).

Integrierte Standardsoftware eignet sich in besonderem Maße als Basis einer ISgestützten Prozeßmessung: Einerseits verfügt SSW über eine hohe Prozeßintegration, wodurch Teilvorgänge im System zu einem Gesamtprozeß horizontal zusammengefügt werden. Andererseits ist in SSW ein hoher Grad an Datenintegration realisiert; Meßpunkte werden in einer integrierten Datenbank gesammelt (Mertens 1995, S. 1-4; Ferstl/Sinz 1993, S. 199). Damit sind zwei entscheidende Voraussetzungen für ein Meßsystem der Prozeßleistungsfähigkeit erfüllt: Eine einheitliche Informationsarchitektur und ihre technische Umsetzung (Eccles 1991, S. 133; Haeckel/Nolan 1993, S. 127). Das prozeßorientierte Meßsystem macht sich die Integration des Informationssystems zunutze, um ein konsistentes Gesamtbild einer Prozeßinstanz zu gewinnen.

Ziel des Nutzenmanagements ist jedoch auch der Vergleich der neuen Prozesse und Systeme mit dem Zustand vor der Inbetriebnahme, der im Regelfall durch die Existenz zahlreicher, nur unzulänglich integrierter Aufgaben, Applikationen und Datenbanken gekennzeichnet ist. Häufig bestehen auch Standardsoftware und Altsysteme nebeneinander (Information Builders 1995, S. 6). Ein Meßsystem für das Nutzenmanagement sollte auch in einer solch heterogenen Umgebung Prozeßdaten liefern können. Voraussetzung ist dafür die Implementierung von Meßsystemen schon in der alten Systemumgebung. Als Implementierungsvorgabe hat sich hier die Basisdatenextraktion bewährt, bei der die Meßsysteme aus den prozeßunterstützenden Datenbanken unverdichtete Basisdaten herausziehen und außerhalb des Transaktionssystems zu Führungsgrößen verdichten, z.B. mit Hilfe von Programmen zur Tabellenkalkulation.

Entscheidend für eine Vergleichbarkeit der Messungen auf der Grundlage verschiedener Informationssysteme ist die semantische Homogenität der Daten (Gassner 1996, S. 12). Diese ist auf Ebene der Basisdaten am ehesten sicherzustellen. Gleichzeitig ermöglichen Basisdaten mit ihrer niedrigen Granularität die größte Vielfalt an Analysen (Gray 1997, S. 83).

Projekterfahrungen im Rahmen des Forschungsprojekts „Process Measurement and Benchmarking“ haben gezeigt, daß sich Meßsysteme nach dem Prinzip der Basisdatenextraktion recht einfach auf andere Systemplattformen, die nicht von Standardsoftware dominiert sind, übertragen lassen. Die Übertragbarkeit im einzelnen ergibt sich hauptsächlich aus der Verfügbarkeit von Meßpunkten im jeweiligen System; wenn beispielsweise keine Zeitstempel in den Datenbanken des Altsystems verzeichnet werden, können Durchlaufzeiten nicht auf Basis von Tagesbruchteilen berechnet werden. Durch die nachträgliche Extraktion auch 
über mehrere Datenquellen hinweg stellte sich eine mangelnde Integration als eher geringes Problem heraus. Der weitgehende Verzicht auf Auswertelogik innerhalb des Extraktionstools erleichtert die Implementierbarkeit auf unterschiedlichen Systemen.

\subsection{Prozeßbenchmarks}

Ein geschäftsorientierter Ansatz zum Nutzenmanagement soll nicht nur durch Vergleich mit dem Ausgangszustand Fortschritte aufzeigen. Noch wichtiger ist eine Abschätzung, inwieweit die erreichbaren Verbesserungspotentiale bereits ausgeschöpft wurden - "People fail to recognize improvement opportunities because they do not know what is possible" (Smith 1996, S. 542). Die Frage, welche Verbesserungen überhaupt möglich sind, ist auch entscheidend für Unternehmen, die ihre generelle Entscheidung für eine Standardsoftware-Einführung von den aufgezeigten Nutzenpotentialen abhängig machen. Eine Antwort kann nur der Einbezug externer Prozeßbenchmarks geben; die maximalen Leistungssteigerungen, die vergleichbare Unternehmen im Zuge ihrer SSW-Einführung verzeichnen konnten, zeigen das Potential für das eigene Unternehmen. Ohne einen Bezug zu externen Leistungsstandards bleiben die Vorgaben für einen Prozeß häufig hinter dem Erreichbaren zurück und damit Unternehmen unter ihren Möglichkeiten (Harrington 1991, S. 219).

In der Umsetzung des Konzepts, die Ableitung von Vorgaben durch externe Benchmarks zu unterstützen, bestehen Defizite. Ein Grund liegt im hohen Aufwand, den die Gewinnung von Benchmarks über Fragebögen verursacht (Schmidt 1992, S. 12). Ein weiterer Grund liegt in der mangelnden Verfügbarkeit glaubwürdiger Zahlen: "Industry standards can sometimes be used to compare one company's performance against another. ... But for most companies, useful figures don't exist. Company and industry performance measures can be very misleading" (Kaydos 1991, S. 124). Die geringe Glaubwürdigkeit resultiert ebenfalls aus der fragebogenbasierten Entstehungsweise von Benchmarks: Viele Befragte geben Schätzungen an, wenn sie eine Antwort nicht wissen (New/Payne 1995, S. 75). Hinzu tritt das Eigeninteresse der Befragten, in einer vergleichenden Studie nicht zu schlecht dazustehen (Chow et al. 1994, S. 22; Wheatley 1998, S. 4).

Abhilfe gegen solche Verzerrungen können objektiv gewonnene Benchmarks schaffen, deren Entstehung, Berechnung und Übertragbarkeit auf das eigene Unternehmen genau nachvollziehbar ist (Hanser 1996, S. 39; Kaydos 1991, S. 124). Ein Weg zu objektiven Benchmarks, die diese Anforderungen erfüllen, liegt in ihrer IS-gestützten Gewinnung. Dabei entstehen die Vergleichswerte aus der Zusammenführung IS-gestützt gewonnener Prozeßdaten aus verschiedenen Unternehmen, die alle die gleiche Software oder zumindest gleichartige Meßsysteme einsetzen. 
Auch hier bietet Standardsoftware wieder eine geeignete Basis. Ein Anbieter von Standardsoftware legt seinen Produkten ein einheitliches Geschäftsmodell zugrunde; die bei den Kunden installierten Systeme unterscheiden sich lediglich graduell durch das sogenannte "Customizing". Messungen weisen damit eine hohe prinzipielle Vergleichbarkeit auf. Zudem erlaubt die hohe Zahl an Anwendern einer bestimmten Standardsoftware die Entwicklung von standardisierten Meßsystemen, die die Vergleichbarkeit weiter steigern. Vor diesem Hintergrund bietet das Institut für Wirtschaftsinformatik der Universität St. Gallen in Zusammenarbeit mit der SAP AG eine Datenbank mit Prozeßbenchmarks für das System R/3 an.

\subsection{Geschäftsnutzen}

Ziel des Nutzenmanagements ist die Messung und Bewertung des Nutzens, den eine Investition in neue Prozesse und Systeme ermöglichte. Aus diesem Grunde spielen in einem letzten Schritt alle bisherigen Komponenten, Prozeßkennzahlen, Meßsysteme und Prozeßbenchmarks, zusammen.

Ein Vergleich zwischen alten und neuen Prozessen und Systemen zeigt den erzielten Nutzen als Leistungssprung in den Führungsgrößen. Ein Vergleich der bestehenden Performance mit externen Benchmarks zeigt ein Nutzenpotential in Form einer Leistungslücke zwischen eigenem und bestem Wert. Setzen die eigenen Prozesse den Standard, besteht z.Z. kein weiteres Verbesserungspotential. Der Einbezug weiterer Vergleichspartner oder die Wiederholung der Messung zu einem späteren Zeitpunkt kann jedoch neue Bestwerte offenbaren.

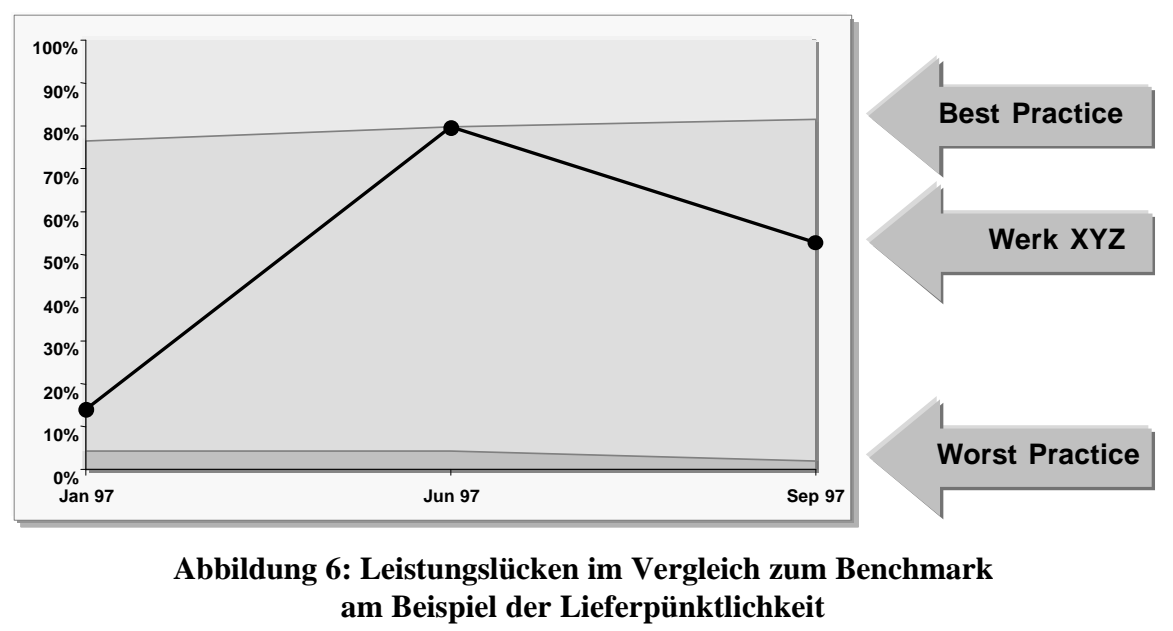

Abb. 6 zeigt für die Lieferpünktlichkeit des bereits in Abb. 3 dargestellten Werks die Leistungslücken, wie sie sich aus dem dreimaligen Vergleich mit externen 
Benchmarks ergaben. Bemerkenswert erscheint die stetige Verbesserung des besten gefundenen Wertes im Laufe der Messungen.

Aus einigen Nutzenpotentialen kann sogar unmittelbar ein finanzieller Vorteil abgeleitet werden. Am Beispiel der Logistikführungsgrösse "Lagerumschlag (Fertigprodukte)" wird dies deutlich (der Lagerumschlag ist in Abb. 5 nicht explizit aufgeführt, geht aber in die Darstellung des "Working Capital" ein). Die Größe gibt an, wie häufig die am Lager verfügbaren Bestände pro Jahr verkauft werden und beschreibt damit die Umschlagshäufigkeit des in Fertigprodukten gebundenen Kapitals, dessen Reduzierung ein zentrales Logistikziel ist (Weber/Kummer 1994, S. 44ff.). Beträgt der durchschnittliche Bestandswert eines Produktes in einem Monat 10 Mio. DM und im gleichen Monat der Wert der Absatzmenge 2 Mio. DM, so errechnen sich daraus ein Lagerumschlag von $(2 * 12) / 10=2,4$ und bei einem Kapitalkostensatz von $8 \%$ monatliche Kapitalbindungskosten in Höhe von $10 * 0.08 / 12=66.666,67$ DM.

Eine Steigerung des Lagerumschlags kann im Rahmen einer StandardsoftwareEinführung mit ihren integrierten Planungsmöglichkeiten beispielsweise durch genauere Absatzprognosen erreicht werden, die dann wiederum bei gleichbleibender Lieferfähigkeit verringerte Sicherheitsbestände erlauben. Eine Steigerung des Lagerumschlags von 2,4 auf 6 bei gleichbleibender Absatzmenge kommt einer Reduzierung des durchschnittlichen Lagerbestands von 10 auf 4 Mio. DM gleich; dies spart monatlich Kapitalbindungskosten in Höhe von 40.000 DM, die als Nutzen direkt dem Projekt zur Standardsoftware-Einführung gutgeschrieben werden können.

Projekte, die im Rahmen einer Standardsoftware-Einführung auch Vertriebs-, Distributions-, Marketing-, Beschaffungs- und Fertigungsprozesse integrieren, können insgesamt Bestandsreduktionen in Höhe von 20\% - 40\% erzielen (Benchmarking Partners 1997b, S. 7). In einem Unternehmen, dessen Prozesse im Zuge der hier beschriebenen Aktivitäten gemessen wurden, reichte bereits eine Senkung der Bestände an Fertigprodukten um 20\% aus, um eine Amortisation des gesamten SSW-Projekts innerhalb von 2 Jahren zu ermöglichen - durch unzureichende Planungsprozesse und mangelnde Transparenz über die verfügbaren Produkte hatte das Unternehmen erhebliche Bestände aufgebaut.

Eine derartig direkte Verbindung zwischen Prozeßverbesserung und finanziellem Nutzen gelingt nur in Ausnahmefällen. Auch wenn Kunden immer wieder die Lieferpünktlichkeit als wichtigstes Kriterium bei ihrer Lieferantenauswahl nennen (s. z.B. Vonderembse et al. 1995, S. 36) und eine gesteigerte Lieferpünktlichkeit insofern Mehrumsätze erwarten läßt, fällt die exakte Berechnung des Zusammenhangs schwer. Größen wie Lieferpünktlichkeit wirken im Regelfall nicht unmittelbar finanziell, sondern eher langfristig über die gesteigerte Kundenzufriedenheit. Dabei konnte zumindest eine positive Korrelation zwischen finanziellem Erfolg und hoher Kundenzufriedenheit verschiedentlich empirisch bewiesen werden (s. die Übersicht bei Griffin et al. 1995). 


\section{Zusammenfassung und Ausblick}

Der dargestellte Ansatz zum Nutzenmanagement mißt und bewertet den Erfolg einer IS/IT-Investition, insbesondere in Standardsoftware, anhand der Prozeßverbesserungen, die durch das Einführungsprojekt erzielt wurden. Damit reagiert der Ansatz auf den Umstand, daß Unternehmen gerade bei der Einführung von Standardsoftware Prozesse und Systeme in einem Zuge umgestalten. Schon die Zurechnung der Projektkosten zu einem der beiden Gestaltungsobjekte fällt schwer; unmöglich erscheint die Trennung positiver Projektergebnisse in organisatorisch und IS-induzierte.

Zur Messung der Prozeßverbesserungen bedient sich der Ansatz eines Katalogs von Prozeßkennzahlen, die mit Hilfe standardisierter Meßsysteme aus den prozeßunterstützenden Informationssystemen extrahiert werden. Eine Implementierung vergleichbarer Meßsysteme auf Standardsoftware und Altsystemen erlaubt den objektiven Vergleich der Zustände vor und nach Inbetriebnahme der neuen Systeme; aus der Zusammenführung von Messungen mehrerer Unternehmen entstehen extern abgesicherte Leistungsstandards.

Die Grenzen dieses Ansatzes liegen insbesondere in den gemessenen Kennzahlen, die sich auf die Erfolgsfaktoren Zeit, Qualität und Flexibilität konzentrieren. Damit gelingt eine Berechnung finanzieller Nutzenpotentiale nur selten. Ein weiterer Begrenzungsfaktor liegt in der Forderung nach IS-gestützter Meßbarkeit der Kennzahlen, die sich nur auf Aufgaben beziehen können, die auch vom Computer erfaßt werden (Österle 1995, S. 178). Wichtige, aber nicht IS-gestützt erhebbare Größen wie die Kundenzufriedenheit können höchstens indirekt z.B. über einen Index gemessen werden, in den IS-gestützt meßbare Größen wie Lieferpünktlichkeit oder Retourenquote als Treiber eingehen (Pieske 1997, S. 45).

Eine logische Fortführung des hier beschriebenen Ansatzes geht von der Messung und Bewertung des Nutzens zur Verbesserung der Prozesse und Informationssysteme, um die Ausschöpfung der Nutzenpotentiale sicherzustellen. Dieser umfassende Ansatz wird als IS-gestütztes Prozeßmanagement bezeichnet und ist in Abb. 7 graphisch dargestellt.

Er begreift noch mehr als die Variante 2 das Verbessern der IS-gestützten Prozesse als fortwährende Aufgabe, die zu inkrementellen Änderungen an Prozessen, Systemen und ihrem Zusammenspiel führt. Das IS-gestützte Prozeßmanagement nutzt Kennzahlen und Meßsysteme aus dem Nutzenmanagement, erweitert sie gegebenenfalls, hilft über detaillierte Schwachstellenanalysen bei der Ableitung von Verbesserungsmaßnahmen und kontrolliert die Umsetzung der Maßnahmen durch kontinuierliche Messungen. 


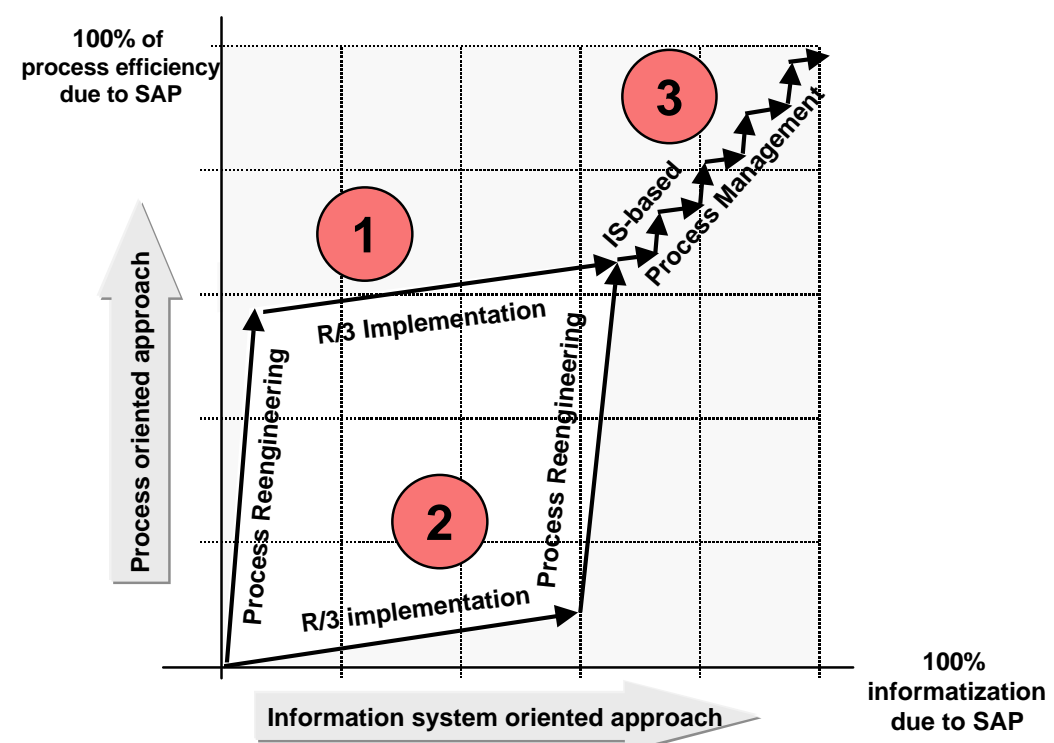

Abbildung 7: IS-gestütztes Prozeßmanagement

\section{Literaturverzeichnis}

Aichele, C. (1997): Kennzahlenbasierte Geschäftsprozeßanalyse. Berlin u.a. 1997.

Aichele, C./Kirsch, J. (1995): Geschäftsprozeßanalyse auf Basis von Kennzahlensystemen, Management \& Computer 3 (1995) 2, S. 123-132

APICS (1996): Most Manufacturers Oblivious to Technology's Payback, The Performance Advantage, Online Edition, 6 (1996) 6, S. 1-3. http://www.lionhrtpub.com/apics/apics-6-96/realworld.html (28.8.1998)

Apostolopoulos, T./Pramataris, K. (1997): Information Technology Investment Evaluation: Investments in Telecommunication Infrastructure, International Journal of Information Management 17 (1997) 4, S. 287-296

Bäckert, W./Brecht, L. (1996): Process Measurement and Benchmarking at Ciba Specialty Chemicals, Vortrag an der SAPPHIRE Europa 1996 (SAP Anwender-Konferenz), Wien 1996

Bäckert, W./Packowski, J. (1997): Business Process Reengineering der europäischen Supply Chain bei der Ciba Specialitätenchemie AG, HMD Theorie und Praxis der Wirtschaftsinformatik 34 (1997) 198, S. 51-65 
Bagchi, P. (1996): Role of benchmarking as a competitive strategy: the logistics experience, International Journal of Physical Distribution \& Logistics Management (1996) 2, S. 4-22

Baumgartner, R. (1995): ABB Customer Focus: ein unternehmerisches Gesamtkonzept, Die Unternehmung (1995) 6, S. 403-409

Becker, M./Fleisch, E./Österle, H. (1997): Business Process Reengineering mit SAP R/3 - Fallstudie Kontron Elektronik, St. Gallen 1997

Benchmarking Partners (1997a): Strategic Partnerships: The Emerging Role of the CIO and CFO, Cambridge, Massachusetts 1997

Benchmarking Partners (1997b): Achieving a Competitive Edge through Enterprise Integration, Cambridge, Massachusetts 1997

Boutellier, R. (1997): Qualität in der Logistik, Management \& Qualität (1997) 9, S. $24-27$

Buxmann, P./König, W. (1996): Organisationsgestaltung bei der Einführung betrieblicher Standardsoftware, Management \& Computer 4 (1996) 3, S. 161-168

Buxmann, P./König, W. (1997): Empirische Ergebnisse zum Einsatz der betrieblichen Standardsoftware SAP R/3, Wirtschaftsinformatik 39 (1997) 4, S. 331-338

Camp, R. (1995): Business Process Benchmarking: Finding and Implementing Best Practices, Milwaukee, Wisconsin 1995

Changchit, Ch./Joshi, K./Lederer, A. (1998): Process and Reality in Information Systems Benefit Analysis, Information Systems Journal 8 (1998) 2, S. 145-162

Chow, G./Heaver, T./Henriksson, L. (1994): Logistics Performance: Definition and Measurement, International Journal of Physical Distribution \& Logistics Management 24 (1994) 1, S. 17-28

Compass (1998): Information Technology Performance Improvement Studies, http://www.compass-analysis.com (27.7.1998)

Computer Finance (1997): Can ERP Project Costs Be Contained?, Computer Finance 7 (1997) 8, S. 1-9

Davenport, T. (1998): Putting the Enterprise into the Enterprise System, Harvard Business Review (1998) July/August, S. 121-131

DeLone, W./McLean, E. (1993): Assessing the Business Value of Information Systems, Arbeitsbericht des David D. Lattanze Centers for Executive Studies in Information Systems at Loyola College in Baltimore, Maryland, http://lattanze.loyola.edu/lattanze/research/wp0493.017.html (27.7.1998)

Dempsey, J./Dvorak, R./ Holen, E./ Mark, D. (1998): A Hard and Soft Look at IT Investments, The McKinsey Quarterly, (1998) 1, S. 126-137 
Dolmetsch, R./Huber, T./Fleisch, E./Österle, H. (1998) Accelerated SAP - 4 Case Studies, St. Gallen 1998

Dusch, M./Möller, M. (1997): Praktische Anwendung der Balanced Scorecard: Ein neuer Ansatz der Fabriksteuerung in der Philips Bildröhrenfabrik Aachen, Controlling (1997) 2, S. 116-121

Eccles, R. (1991) The Performance Measurement Manifesto, Harvard Business Review (1991) Jan/Feb., S. 131-137

Edmondson, G. (1997): Silicon Valley on the Rhine, Business Week, (1997) 3. Nov., S. 40-47

Farbey, B./Land, F./Targett, D. (1995): A Taxonomy of Information Systems Applications: The Benefits' Evaluation Ladder, European Journal of Information Systems (1995) 4, S. 41-50

Ferstl, O./Sinz, E. (1993): Grundlagen der Wirtschaftsinformatik, Band 1, München u.a. 1993

Forrester (1996): The Prudent Approach to R/3, Cambridge, Massachusetts 1996

Fortuin, L. (1988): Performance indicators-why, where and how?, European Journal of Operational Research 34 (1988) 1, S. 1-9

Gassner, C. (1996): Konzeptionelle Integration heterogener Transaktionssysteme, St. Gallen 1996

Gray, P. (1997): Mining for data warehousing gems, Information Systems Management 14 (1997) 4, S. 82-86

Griffin, A./Gleason, G./Preiss, R./Shevenaugh, D. (1995): Best Practice for Customer Satisfaction in Manufacturing Firms, Sloan Management Review 36 (1995) 2, S. 87-98

Groenfeldt, T. (1997): Who's in the Driver's Seat?, Journal of Business Strategy 18 (1997) 1, S. 37-41

Grover, V./Jeong, S./Teng, J. (1998): Survey of Reengineering Challenges, Information Systems Management 15 (1998) 1, S. 53-59

Haeckel, S./Nolan, R. (1993): Managing by wire, Harvard Business Review (1993) Sept/Okt, S. 122-132

Hanser, P. (1996): Benchmarking - Von den Besten lernen, Absatzwirtschaft (1996) 2, S. 32-41

Harrington, H. (1991): Business Process Improvement, New York 1991

Horváth, P. (1997): Die "Vorderseite" der Prozeßorientierung, Controlling 9 (1997) 2, S. 114

Information Builders (1995): Data Migration to SAP R/3, New York 1995

Jurison, J. (1997): Reevaluating Productivity Measures, Information Systems Management 14 (1997) 1, S. 30-34 
Kaplan, R./Norton, D. (1993): Putting the Balanced Scorecard to Work, Harvard Business Review (1993) Sep/Oct, S. 134-147

Kaydos, W. (1991): Measuring, Managing, and Maximising Performance: What Every Manager Needs to Know about Quality and Productivity to Make Real Improvements in Performance, Cambridge, Massachusetts 1991

Kempis, R./Ringbeck, J. (1998): Manufacturing's Use and Abuse of IT, The McKinsey Quarterly (1998) 1, S. 138-150

Kidler, B. (1998): TCO - Am Anfang stand der Kundennutzen? Lowering TCO für Geschäftsprozesse, IM - Information Management \& Consulting 13 (1998) 2, S. 24-27

Kidler, B./Gümbel, H. (1998): Lowest Cost of Ownership, Vortrag an der SAPPHIRE Europa 1998 (SAP Anwender-Konferenz), http://www.sap.com/ sapphire/madrid/pdf/b08.pdf (11.9.1998)

Knolmayer, G./von Arb, R./Zimmerli, Chr. (1997): Erfahrungen mit der Einführung von SAP R/3 in Schweizer Unternehmen, Bern 1997

Lacity, M./Hirschheim, R. (1994): The Role of Benchmarking Services in Demonstrating I.S. Effectiveness to Senior Management, Proceedings of the Second European Conference on Information Systems (Part IV), Nijenrode 1994

Martin, M. (1998): An ERP Strategy, Fortune Magazine (1998) 2, S. 95-97

McMullan, A. (1996): Supply Chain Management in Asia Pacific Today, International Journal of Physical Distribution \& Logistics Management 26 (1996) 8, S. 79-95

Mende, M. (1995): Ein Führungssystem für Geschäftsprozesse, St. Gallen 1995

Mertens, P. (1995): Integrierte Informationsverarbeitung 1 - Administrationsund Dispositionssysteme in der Industrie, Wiesbaden 1995

Meyer, C. (1994): How the Right Measures Help Teams Excel, Harvard Business Review (1994) May/June, S. 95-103

Millen, R./Maggard, M. (1995): The change in quality practices in logistics: 1995 versus 1991, Total Quality Management 8 (1997) 4, S. 173-179

Muschter, S. (1999): IS-gestütztes Prozeßmanagement, St. Gallen 1999

New, S./Payne, P. (1995): Research Frameworks in Logistics - Three Models, Seven Dinners and a Survey, International Journal of Physical Distribution \& Logistics Management 25 (1995) 10, S. 60-77

Nippel, H. (1995): Qualitätsmanagement in der Logistik, Wiesbaden 1995

o.V. (1998): Baan ist 1997 ein gutes Stück weitergekommen, Frankfurter Allgemeine Zeitung (1998) 4. Feb., S. 23

Österle, H. (1995): Business Engineering: Prozeß- und Systementwicklung, Band 1 - Entwurfstechniken, Berlin u.a. 1995 
Pieske, R. (1997): Jeder reagiert anders - Alternative Vorgehensweisen zur Ermittlung der Kundenzufriedenheit, io management, (1997) 7/8, S. 4047

Potthof, I. (1998): Empirische Studien zum wirtschaftlichen Erfolg der Informationsverarbeitung, Wirtschaftsinformatik 40 (1998) 1, S. 54-65

Rehäuser, J./Krcmar, H. (1995): Benchmarking im Informationsmanagement als Instrument eines umfassenden IV-Controlling, DV-Management (1995) 3, S. $107-112$

Reichmann, T. (Hrsg.) (1993): DV-gestütztes Unternehmens-Controlling: Kennzahlengestütztes Logistik-Controlling, München 1993

Rieker, J. (1998): Unternehmen SAP - Die drei von der Baustelle, Manager Magazin 28 (1998) 4, S. 114-126

Riepl, L. (1998): TCO versus ROI, IM - Information Management \& Consulting 13 (1998) 2, S. 7-12

SAP (1998a): SAP AG 1997 Sales Rise 62\% To DM 6.02 Billion, Presseerklärung der SAP AG vom 27. 1.1998, Walldorf 1998

SAP (1998b): Corporate Profile der SAP AG, http://www.sap.com (15.7.1998)

Schmidt, J. (1992): The Link between Benchmarking and Shareholder Value, Journal of Business Strategy 3 (1992) 2, S. 7-13

Skyrme, D./Amidon, D. (1998): New Measures of Success, Journal of Business Strategy 19 (1998) 1, S. 20-24

Smith, G. (1996): Identifying quality problems: Prospects for improvement, Total Quality Management 7 (1996) 5, S. 535-552

Stewart, T. (1997): Owens Corning - Back From the Dead, Fortune Magazine (1997) 10, S. 78-82

Tushman, M./O’Reilly, C. (1996): The Ambidextous Organizations: Managing Evolutionary and Revolutionary Change, California Management Review 38 (1996) 4, S. 8-30

Venkatraman, N./Ramanujam, V. (1986): Measurement of Business Performance in Strategy Research: A Comparison of Approaches, Academy of Management Review 11 (1986) 4, S. 801-814

Vonderembse, M./Tracey, M./Tan, Ch./Bardi, E. (1995): Current Purchasing Practices and JIT, International Journal of Physical Distribution \& Logistics Management 25 (1995) 3, S. 33-48

Ward, J./Taylor, P./Bond, P. (1996): Evaluation and Realisation of IS/IT Benefits: An Empirical Study of Current Practice, European Journal of Information Systems (1996) 4, S. 214-225

Weber, J. (Hrsg.) (1995): Kennzahlen für die Logistik, Stuttgart 1995 
Weber, J./Kummer, S./Grossklaus, A./Nippel, H. (1997): Methodik zur Generierung von Logistik-Kennzahlen, Betriebswirtschaftliche Forschung und Praxis 49 (1997) 4, S. 438-454

Weber, J./Kummer, S. (1994): Logistikmanagement - Führungsaufgaben zur Umsetzung des Flussprinzips im Unternehmen, Stuttgart 1994

Wheatley, M. (1998): Benchmarking - Follow the Leader, CIO Magazine (1998) 1. Juli, S. 1-8 